sota. Calculations were completed at Wright Air Development Center, WrightPatterson Air Force Base, Ohio. The author expresses his thanks to Dr. Ernest T. Parker for suggesting the problem, to Remington Rand UNIVAC and to the Digital Computation Branch at Wright Air Development Center for providing time on their UNIVAC Scientific computers.

Wright Air Development Center

Wright-Patterson Air Force Base, Ohio

1. H. K. NANDI, "A further note on non-isomorphic solutions of incomplete block designs," Sankhya, v. 7, 1945-46, p. 313-316.

2. John RIORda , An Introduction to Combinatorial Analysis, John Wiley \& Sons, Inc., New York, 1958, p. 51 .

3. H. J. RYSER, "Geometries and incidence matrices," Amer. Math. Mon., v. 62, 1955, p. 25-31.

\title{
On the Numerical Treatment of Heat Conduction Problems with Mixed Boundary Conditions
}

\section{By Arnold N. Lowan}

Abstract. The two-dimensional problem of heat conduction in a rectangle where the temperature is prescribed over a portion of the boundary while the temperature gradient is prescribed over the remainder of the boundary, may be treated numerically by replacing the differential equation of heat conduction and the equations expressing the given initial and boundary conditions by their difference analogs

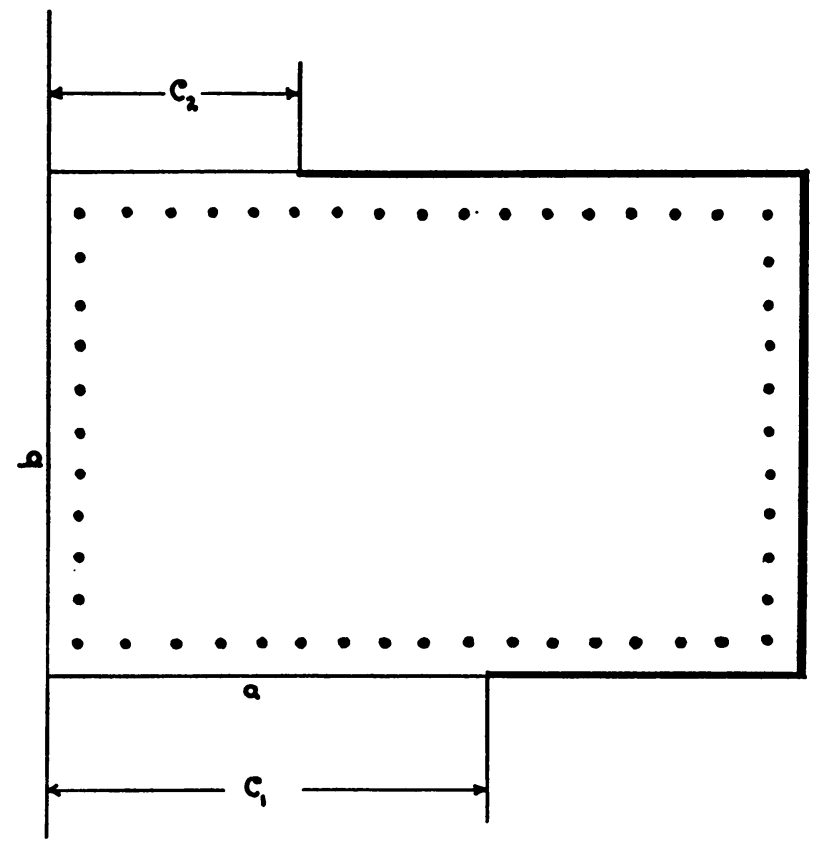

FIG. 1.-Rectangular domain with "mixed" boundary conditions.

Received September 15, 1959. 
and solving the resulting system. It is shown that if the scheme is to be stable the intervals $\Delta x$ and $\Delta y$ must be chosen so that $k \Delta t /(\Delta x)^{2}+k \Delta t /(\Delta y)^{2} \leqq \frac{1}{2}$.

Consider the two-dimensional problem of heat conduction in a rectangle, Figure 1 , when the temperature is prescribed over the thin line portion of the boundary, while the temperature gradient is prescribed over the heavy line portion of the boundary. This is a typical problem with "mixed" boundary conditions and should not be confused with the considerably simpler problem when the temperature is prescribed over certain complete sides of the rectangle, while the temperature gradient is prescribed over the remaining sides. As far as the writer is aware no analytical solution of the mixed boundary value problem above formulated (or of the analogous problem for the cylinder) is to be found in the literature. We must therefore (if interested in numerical answers) resort to the alternative of substituting for the differential equation of heat conduction and for the equations expressing the initial and boundary conditions their appropriate difference analogs, and solving the resulting system.

The mathematical formulation of the problem is as follows:

$$
\begin{array}{cc}
\frac{\partial T}{\partial t}= & k\left(\frac{\partial^{2} T}{\partial x^{2}}+\frac{\partial^{2} T}{\partial y^{2}}\right) \quad 0 \leqq x \leqq a, 0 \leqq y \leqq b, t>0 \\
T(x, y, 0)=f(x, y) & 0 \leqq y \leqq b \\
T(0, y, t)=0 & 0 \leqq x \leqq c_{1} \\
T(x, 0, t)=0 & c_{1} \leqq x \leqq a \\
{\left[\frac{\partial}{\partial y} T(x, y, t)\right]_{y=0}=0} & 0 \leqq y \leqq b \\
{\left[\frac{\partial}{\partial x} T(x, y, t)\right]_{x=a}=0} & \\
\left.\left[\frac{\partial}{\partial y} y, t\right)\right]_{y=b}=0 & c_{2} \leqq x \leqq a \\
T(x, b, t)=0 & 0 \leqq x \leqq c_{2}
\end{array}
$$

where for the sake of simplicity we have at first assumed that the prescribed temperature and temperature gradient are $=0$. The difference analogs of the above equations are:

$$
\begin{array}{rr}
T_{h, k, n+1}=\beta T_{h, k-1, n}+\alpha & T_{h-1, k, n}+(1-2 \alpha-2 \beta) T_{h, k, n} \\
+\alpha T_{h+1, k, n}+\beta T_{h, k+1, n} \quad h=1,2,3, \cdots M, k=1,2,3 \cdots N \\
T_{h, k, 0}=f(h \Delta x, k \Delta y) \\
T_{0, k, n}=0 & 1 \leqq k \leqq N \\
T_{h, 0, n}=0 & 1<h<c_{1} / \Delta x \\
T_{h, 1, n}=T_{h, 0, n} & c_{1} / \Delta x \leqq h \leqq a / \Delta x
\end{array}
$$




$$
\begin{aligned}
T_{M+1, k, n} & =T_{M, k, n} \\
T_{h, N+1, n} & =T_{h, N, n} \\
T_{h, N+1, n} & =0
\end{aligned}
$$$$
1 \leqq k \leqq N
$$$$
c_{2} / \Delta x \leqq h \leqq a / \Delta x
$$$$
1 \leqq h \leqq c_{2} / \Delta x
$$

where

$$
\begin{gathered}
T_{h, k, n,}=T(h \Delta x, k \Delta y, n \Delta t) ; \quad \alpha=\frac{k \Delta t}{(\Delta x)^{2}} ; \quad \beta=\frac{k \Delta t}{(\Delta y)^{2}} \\
\Delta x=a /(M+1) \quad \text { and } \quad \Delta y=b /(N+1) .
\end{gathered}
$$

It will be convenient to consider the $M N$ temperatures $T_{h, k, n}$ with $h=1,2,3, \cdots$ $M$ and $k=1,2,3, \cdots N$ as the components of an $M \times N-$ dimensional vector to be denoted by $\mathbf{T}_{n}$. It will also be convenient to replace the two subscripts $h$ and $k$ identifying the lattice point $P_{h k}$ (i.e., the point with coordinate $x=h \Delta x$ and $y=$ $k \Delta y$ ) by the single subscript $p$ running from $p=1$ to $p=M N$ with the understanding that for the $M$ lattice points corresponding to $k=1, p$ runs from 1 to $M$, for the next set of $M$ lattice points corresponding to $k=2 p$ runs from $M+1$ to $2 M, \cdots$ etc. The system of $M N$ equation $\left(1^{*}\right)$ may then be written in the matrixvector form

$$
\mathbf{T}_{n+1}=A \mathbf{T}_{n} .
$$

As is well known, to prove the stability of (9), it suffices to prove that if $S$ denotes the largest of the sums of absolute values of the elements of the rows of $A$ then $S \leqq 1$.

Let $\Omega$ denote the set of lattice points closest to the boundary. It is clear that if the difference equation $\left(1^{*}\right)$ is applied to lattice points lying inside of $\Omega$, the resulting equation (which is in fact equation $\left(1^{*}\right)$ itself) has the five non-vanishing coefficients $\beta, \alpha, 1-2 \alpha-2 \beta, \alpha$ and $\beta$. If we assume $1-2 \alpha-2 \beta \geqq 0$ or $\alpha+\beta \leqq \frac{1}{2}$ it is clear that the sum of the absolute values of the coefficients is $=1$. We shall show that if $\left(1^{*}\right)$ is applied to lattice points belonging to the set $\Omega$, the resulting equation may be characterized by the fact that the sum of absolute values of the coefficients is smaller than unity. Consider for instance the form taken by $\left(1^{*}\right)$ when applied to a point of $\Omega$ such that $h \Delta x \leqq c_{1}$. Since for such a point $T_{h, 0}=0$ the resulting equation has the four non-vanishing coefficients $\alpha, 1-2 \alpha-2 \beta, \alpha$ and $\beta$. If again we assume $1-2 \alpha-2 \beta>0$, it follows that the sum of the absolute values of the coefficients is $=1-\beta<1$. In an entirely similar manner it is shown that if $\left(1^{*}\right)$ is applied to lattice points in $\Omega$ for which $h \Delta x \geqq c_{2}$ and the boundary condition $\left(5^{*}\right)$ is taken into account, the sum of the absolute values of the coefficients of the resulting equation is $1-\alpha<1$. Similar conclusions may be drawn in the case of all lattice points in $\Omega$. Thus the quantity $S$ previously defined is $=1$. The stability of the difference scheme under consideration is thus proven, provided that the intervals $\Delta x$ and $\Delta t$ are chosen so that

$$
\alpha+\beta=\frac{k \Delta t}{(\Delta x)^{2}}+\frac{k \Delta t}{(\Delta y)^{2}} \leqq \frac{1}{2} .
$$

In the above discussion we assumed that the prescribed temperature is $=0^{\circ} \mathrm{C}$ on the thin line portion of the boundary. If instead, nonvanishing temperatures are 
prescribed on this portion, the criterion of stability is the same as before, since the error vector satisfies $\left(1^{*}\right)$ and evidently is $=0$ on the portion of the boundary in question.

We shall now discuss the modifications in the above analysis if the prescribed temperature gradient does not vanish. Let the above boundary conditions (5), (6) and (7) be replaced by

$$
\begin{array}{ll}
\frac{\partial T}{\partial y}=\phi_{1}(x, t) & c_{1} \leqq x \leqq a, y=0 \\
\frac{\partial T}{\partial x}=\Phi(y, t) & x=a, 0 \leqq y \leqq b \\
\frac{\partial T}{\partial y}=\phi_{2}(x, t) & c_{2} \leqq x \leqq a, y=b .
\end{array}
$$

The difference analogs of the last three equations are

$$
\begin{array}{rlrl}
T_{h, 0, n} & =T_{h, 1, n}+\Delta y \cdot \phi_{1}(h \Delta x, n \Delta t) & c_{1} / \Delta x \leqq h \leqq a / \Delta x \\
T_{M+1, k, n}=T_{M, k, n}-\Delta x \cdot \Phi(k \Delta y, n \Delta t) & 1 \leqq k \leqq N \\
T_{h, N+1, n}=T_{h, N, n}-\Delta y \cdot \phi_{2}(h \Delta x, n \Delta t) & c_{2} / \Delta x \leqq h \leqq a / \Delta x
\end{array}
$$

If the difference equation $\left(1^{*}\right)$ is applied to the lattice points for which the last three equations hold, we ultimately get

$$
\begin{gathered}
T_{h, 1, n+1}=\alpha T_{h-1,1, n}+(1-2 \alpha-2 \beta) T_{h, 1, n}+\alpha T_{h+1,1, n} \\
+\beta T_{h, 2, n}+U_{h, 1, n} \quad c_{1} / \Delta x \leqq h \leqq a / \Delta x \\
\begin{aligned}
& T_{M, k, n+1}=\beta T_{M, k-1, n}+\alpha T_{M-1, k, n}+(1-2 \alpha-2 \beta) T_{M, k, n} \\
&+\beta T_{M, k+1, n}+U_{M, k, n} \quad 1 \leqq k \leqq N \\
& T_{h, N, n+1}=\beta T_{h, N-1, n}+\alpha T_{h-1, N, n}+(1-2 \alpha-2 \beta) T_{h, N, n} \\
&+T_{h+1, N, n}+U_{h, N, n}
\end{aligned}
\end{gathered}
$$

where we have put

$$
\begin{array}{rlrl}
U_{h, 1, n} & =\beta \Delta y \cdot \phi_{1}(h \Delta x, n \Delta t) & c_{1} / \Delta x \leqq h \leqq a / \Delta x \\
U_{M, k, n}=-\alpha \Delta x \cdot \Phi(k \Delta y, n \Delta t) & 1 \leqq k \leqq N \\
U_{h, N, n}=-\beta \Delta y \cdot \phi_{2}(h \Delta x, n \Delta t) & c_{2} / \Delta x \leqq h \leqq a / \Delta x .
\end{array}
$$

The system of $M N$ equations obtained by applying $\left(1^{*}\right)$ to the $M N$ lattice points may be written in the form

$$
\mathrm{T}_{n+1}=A \mathrm{~T}_{n}+\mathrm{U}_{n}
$$

where $U_{n}$ is a vector whose non-vanishing components are defined in (16) and whose remaining components are $=0$. Since the error vector $\mathbf{E}_{n}$ satisfies the difference equation (17) it is readily seen that 


$$
\mathrm{E}_{n+1}=A^{n} \mathrm{E}_{0}+A^{n-1} \mathrm{U}_{0}+A^{n-2} \mathrm{U}_{1}+\cdots+\mathrm{U}_{n-1} .
$$

From (18) it follows at once that the criterion for the stability of (17) is identical with that for (9), namely that $\Delta x$ and $\Delta y$ must be chosen so that $\alpha+\beta \leqq \frac{1}{2}$.

It may be briefly mentioned that the above analysis may be extended to the more general case of the boundary conditions $p T+q(\partial T / \partial n)=F(t)$ where $p$ and $q$ take on prescribed values along the boundary. It may also be mentioned that the above analysis may be extended to problems with cylindrical and spherical symmetry.

Yeshiva University

New York, New York: and

AVCO Corporation

Wilmington, Massachusetts

\section{High Precision Calculation of Arcsin $x, \operatorname{Arccos} x$, and $\operatorname{Arctan} x$}

\section{By I. E. Perlin and J. R. Garrett}

1. Introduction. In this paper a polynomial approximation for Arctan $x$ in the interval $0 \leqq x \leqq \tan \pi / 24$, accurate to twenty decimal places for fixed point routines, and having an error of at most 2 in the twentieth significant figure for floating point routines is developed. By means of this polynomial Arctan $x$ can be calculated for all real values of $x$. Arcsin $x$ and $\operatorname{Arccos} x$ can be calculated by means of the identities:

$$
\operatorname{Arctan} \frac{x}{\sqrt{1-x^{2}}}=\operatorname{Arcsin} x=\frac{\pi}{2}-\operatorname{Arccos} x
$$

2. Polynomial Approximation for Arctan $x$. A polynomial approximation for the Arctangent is obtained from the following Fourier series expansion, given by Kogbetliantz [1], [2] and Luke [3].

$$
\operatorname{Arctan}(x \tan 2 \theta)=2 \sum_{i=0}^{\infty} \frac{(-1)^{i}(\tan \theta)^{2 i+1}}{2 i+1} T_{2 i+1}(x)
$$

where $T_{i}(x)$ are the Chebyshev polynomials, i.e., $T_{i}(\cos y)=\cos (i y)$. The expansion (2.1) is absolutely and uniformly convergent for $|x| \leqq 1$ and $0 \leqq \theta<\pi / 4$. Thus,

An approximating polynomial is obtained by truncating (2.1) after $n$ terms.

$$
P(x \tan 2 \theta)=2 \sum_{i=0}^{n-1} \frac{(-1)^{i}(\tan \theta)^{2 i+1}}{2 i+1} T_{2 i+1}(x) .
$$

The truncation error is

$$
\left|\epsilon_{T}\right| \leqq \tan 2 \theta(\tan \theta)^{2 n}|x| .
$$

Received December 11, 1959; in revised form, February 16, 1960. The work reported in this paper was sponsored by the Air Force Missile Development Center, Alamogordo, New Mexico. 